\title{
Plant Responses Underlying Timely Specialized Metabolites Induction of Brassica Crops
}

\author{
Maroua Doghri1,2, Víctor Manuel Rodríguez ${ }^{1}$, Daniel J. Kliebenstein ${ }^{3,4}$ and \\ Marta Francisco ${ }^{1 *}$
}

${ }^{1}$ Misión Biológica de Galicia (MBG-CSIC), Pontevedra, Spain, ${ }^{2}$ Department of Plant Biology, Faculty of Biology, Institute of Biotechnology and Biomedicine, University of Valencia, Valencia, Spain, ${ }^{3}$ Department of Plant Sciences, University of California, Davis, Davis, CA, United States, ${ }^{4}$ DynaMo Center of Excellence, University of Copenhagen, Frederiksberg, Denmark

\section{OPEN ACCESS}

Edited by:

Jose Antonio Monreal, University of Seville, Spain

Reviewed by:

Melissa Hamner Mageroy, Norwegian Institute of Bioeconomy Research (NIBIO), Norway

Atsushi Fukushima, Kyoto Prefectural University, Japan

*Correspondence: Marta Francisco mfrancisco@mbg.csic.es

Specialty section: This article was submitted to Plant Metabolism and Chemodiversity, a section of the journal

Frontiers in Plant Science

Received: 02 November 2021 Accepted: 30 December 2021 Published: 03 February 2022

Citation:

Doghri M, Rodríguez VM, Kliebenstein DJ and Francisco M (2022) Plant Responses Underlying

Timely Specialized Metabolites

Induction of Brassica Crops.

Front. Plant Sci. 12:807710.

doi: 10.3389/fp/s.2021.807710
A large subset of plant stress-signaling pathways, including those related with chemical defense production, exhibit diurnal or circadian oscillations. However the extent to which diurnal or circadian time influences the stress mediated accumulation of plant specialized metabolites remains largely unknown. Because plant responses to physical stress (e.g., wounding) is considered a common component of mounting a response against a broad range of environmental stresses, including herbivory, we have utilized mechanical wounding as the stress stimulus to determine the direct contribution of time of day on the induced defenses of Brassica crops. We analyzed glucosinolates (GSLs) from leaves of broccoli (Brassica oleracea) and turnip greens (Brassica rapa) following exposure to mechanical wounding at dawn (ZTO), mid-day (ZT4), and dusk (ZT8). Several GSLs differentially accumulated and their changes depended upon the time of day at wounding was performed. This response varied considerably between species. In a parallel experiment, we investigated whether diurnal activation of Brassica phytochemicals in response to wounding might prime plants against herbivore attack. Results showed that maximal response of plant chemical defense against larvae of the generalist pest Mamestra brassicae occurred at ZTO in broccoli and ZT8 in turnip greens. Metabolome analysis for global trends of time dependent compounds showed that sulfur-containing phytochemicals, GSL hydrolysis products, auxin-signaling components, and other metabolites activators of plant disease resistance (nicotinamide and pipecolate) had important contributions to the responses of $M$. brassicae feeding behavior in broccoli at morning. Overall, the findings in this study highlight a significant role for time of day in the wound stress responsive metabolome, which can in turn affect plant-herbivore interactions.

Keywords: Brassicaceae, timely induced resistance, herbivory, Mamestra brassicae, wounding, metabolomics

\section{INTRODUCTION}

Plants are constantly constrained by a wide array of biotic and abiotic stresses, of which damage caused by chewing insects greatly affects plant performance and survival. To defend themselves, plants have evolved a sophisticated defensive system allowing them to perceive mechanical injury and trigger an induced defensive response. Wounding in the plant tissue and/or herbivore feeding 
cues invokes a reprogramming of defense-related gene expression including genes required for de novo biosynthesis of herbivoreinduced chemical defenses (Savatin et al., 2014; Jacobo-Velázquez et al., 2015; Body et al., 2019; Guan et al., 2021). An example of this are the glucosinolates (GSLs), a specific class of secondary compounds present in plants of the Brassicaceae family that provide resistance to generalist herbivores. This defensive system imposes an energetic and metabolic cost to the plant since energy and resources are diverting away from other plant processes such as growth or primary metabolism toward generating these specialized compounds (Agrawal et al., 2002; Paul-Victor et al., 2010). Thus, it is crucial for the plant to sacrifice this investment only when it is necessary.

One mechanism that dynamically adjusts plant overall metabolism in anticipation of a highly predictable environmental cues is the circadian clock. The circadian clock is an endogenous timekeeper that orchestrates rhythmic behaviors of physiological, metabolic and developmental processes in consonance with external cyclic events (McClung, 2019). In plants, the circadian clock is crucial to cope with daily fluctuations of light intensity, temperature and humidity. In addition, herbivores, pathogens and pollinators rely on endogenous clocks and therefore represent predictable biotic threats. Several lines of evidence indicate that large subsets of biotic and abiotic defense-signaling pathways in plants are clock regulated. Genes from defense pathways show rhythmic expression and their transcriptional response often depends on the time of the day at which the plants are exposed to the stress (Bhardwaj et al., 2011; Wang et al., 2011; Zhang et al., 2013). For instance, Walley et al. (2007) showed that wound responsive genes, inducing both biotic and abiotic stress responses, are circadian regulated with consolidated phases of peak expression at certain times of the day. In concordance, the production of wound-induced phytohormones such as Jasmonic acid (JA) and Salicylic acid (SA), as well as downstream activation of plant defense metabolites, including GSLs, is also dependent on circadian periodicity (Covington et al., 2008; Goodspeed et al., 2012, 2013; Soengas et al., 2018a,b; Lei et al., 2019).

It has been hypothesized that plants utilize the clock as a strategy to anticipate the timing of potential threats to phase basal defenses to the particular time of the day when they are most likely to be needed (Harmer et al., 2000; Goodspeed et al., 2012; Burow and Halkier, 2017). However, to date there is only a handful of studies addressing the circadian connection between plant defense signaling pathways and the timing of pest attack. Goodspeed et al. (2012), reported that Arabidopsis resistance against the generalist herbivore insect Trichoplusia ni, is highly dependent on clock-regulated diurnal accumulation of endogenous JA. In a complementary experiment, they demonstrated that cyclic activation of GSLs is required for this phase-dependent resistance (Goodspeed et al., 2013). Consistently, Lei et al. (2019) and Lei and Zhu-Salzman (2021) reported that the link between the circadian clock and plant defense against green peach aphids Myzus persicae is mediated by the cyclic expression pattern of key indole GSL biosynthetic genes. It is well known that wounding or herbivory often induces JA levels and in consequence GSLs compared with their basal levels (van Dam et al., 2004; Jansen et al., 2009).
However, to what extent the dimension of time and/or the circadian clock contribute to the synthesis and mobilization of plant induced resistant compounds under stress responses remains largely unknown.

The aim of this work is to begin providing more insight into how temporal dynamics influence the synthesis and mobilization of induced chemical defenses and if temporal variability in defensive response may alter future rates of herbivory. To start to solve these questions, this work studied how wounding stress is influenced by crosstalk with the diurnal cycle in two different crop species of the Brassicaceae family, broccoli (B. oleracea) and turnip greens (B. rapa). In particular, we first examined plant defense capacity (measured in terms of GSL content) in response to wounding stress, and included "time of day" as a controlled factor. Our work revealed that induction of plant defensive compounds in response to a repetitive damage exerted by wounding depends on the time of the day at which wounding takes place and this response differed between species. We further demonstrated that timely activation of Brassica crops phytochemicals by wounding might prime plants against future herbivore attack. Global metabolome analysis of leaves harvested at the time of maximal resistance against the generalist pest Mamestra brassicae indicated that timely induced sulfurcontaining phytochemicals, GSLs breakdown products, auxinsignaling components, and other metabolites activators of plant resistance such as nicotinamide and pipecolate are behind this response. Together, these data highlight a significant role for time of day in the induced plant defensive compounds affecting plant-herbivore interactions.

\section{MATERIALS AND METHODS}

\section{Plant Growth and Harvest Conditions}

Seeds of a commercial hybrid of broccoli "green calabrese" (B. oleracea var. botrytis) (Batlle S.A., Barcelona) and a local variety of turnip greens "globo blanco de Lugo" (B. rapa ssp. rapa) (WAM S.L.L, Pontevedra) were planted in potting soil stratified at $4^{\circ} \mathrm{C}$ in the dark for 3 days to optimize germination. Plants were sown in a randomized block design consisting of 72 pots $(4 \times 4 \mathrm{~cm})$ per genotype, each containing one plant. All plants were grown in short-day conditions $(8 / 16 \mathrm{~h}$ light/dark) in a growth chamber under controlled conditions $\left(20^{\circ} \mathrm{C}\right.$ of temperature and $80 \%$ of humidity). Because it has been hypothesized that plant responses to physical stress is a mechanism common to mounting a response against a broad range of environmental stresses, including herbivory (Walley et al., 2007), we utilized mechanical wounding as the stress stimulus. To study how time of day effects plant induced resistance in response to wounding, sampling and treatments were performed at various Zeitgeber times (ZT) (Figure 1). At the five-leaf stage, each pool of plants $[12$ plants $\times$ treatment (wounded and control) $\times$ time-point $\times$ genotype] were exposed to mechanical wounding simulating three different day-time attacks: dawn (ZT0), mid-day (ZT4), and dusk (ZT8) during three consecutive days (indicated in Figure 1). For wounding treatments, we used laboratory forceps damaging the third 


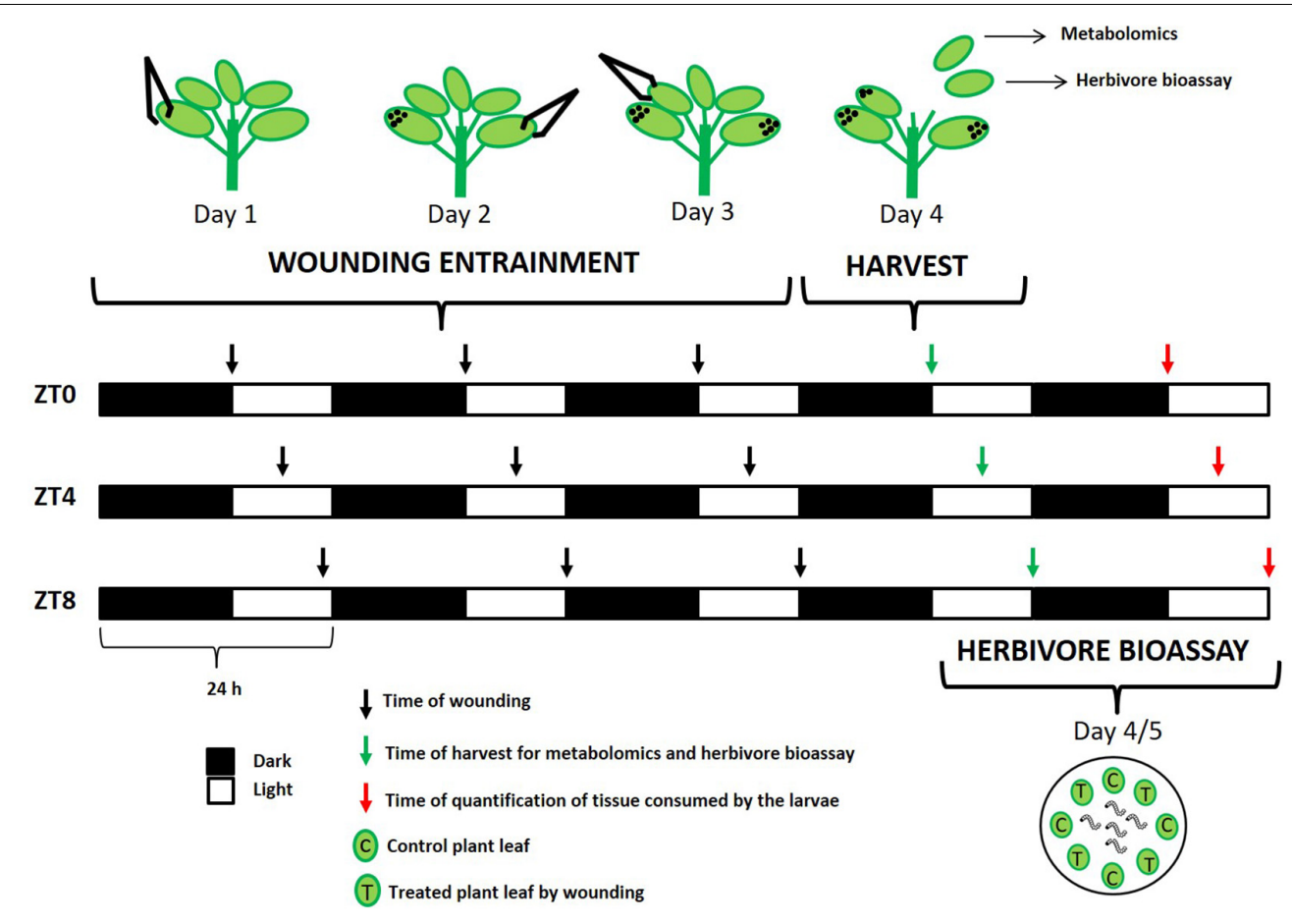

FIGURE 1 | Scheme of the wounding treatment at different time points (ZTO, dawn; ZTO, mid-day; ZT8, dusk). Plants were wounded during three consecutive days and harvested at fourth day. Black arrows represent the time of day at which wounding was performed. Green arrows represent the time of day at which leaves were harvested for metabolomics and herbivore bioassay. Red arrow represents the time of day at which quantification of tissue consumed by the larvae was done for each experiment. Black dots on the leaves indicate previously wounding leaves.

superior part of the leaf, which effectively wounded $\sim 30 \%$ of the leaf area. One leaf per day was wounded starting from the oldest. For the control treatment, plants were kept unwounded. On the fourth day, at the same time-point that each pool of plants was trained to receive damage (ZT0, ZT4, and ZT8) the two youngest leaves per plant were harvested from each genotype and treatment. One leaf was used for metabolite analysis and the other one was used for feeding experiments. For metabolite analysis, individual leaves were cut, placed in plastic scintillation vials and immediately frozen in liquid nitrogen and stored at $-80^{\circ} \mathrm{C}$. Frozen samples were lyophilized and ground to a fine powder using an electric mill and stored in tubes until extraction and analysis.

\section{Insects}

Mamestra brassicae eggs were supplied by the Centre de Recherche de Versailles (Institut National de la Recherche Agronomique, France) and incubated at $20^{\circ} \mathrm{C}$ until they hatched. Neonate larvae were maintained on cabbage leaves under laboratory conditions until the bioassays were set up. To test whether the larvae feeding activity is affected by the light/dark cycle, eight petri dishes containing three leaf-disks of broccoli and turnip greens (separately) were placed with three $M$. brassicae larvae. Larvae were entrained to $12 \mathrm{~h}$ light $12 \mathrm{~h}$ dark cycles for 2 days prior to testing. The remaining leaf area after insect feeding was recorded at the end of the light period and the end of the dark period during two consecutive days. The leaf area in each experiment was measured using the software ImageJ (Schneider et al., 2012). We found that M. brassicae larvae feed similarly during light and dark period under constant temperature conditions (data not shown).

\section{Herbivore Bioassay}

For feeding experiments, three disks of $2 \mathrm{~cm}$ diameter from control and treated leaves were placed in alternate order in a Petri dish along with five two-instar larvae of $M$. brassicae L. (Noctuidae) (Figure 1). We used four replicates for each treatment per genotype at each time-point. Petri dishes were placed in the growth chamber under the same environmental conditions used for plant growth. Larvae were allowed to eat during $24 \mathrm{~h}$. We recorded pictures of each disk using a digital camera (Nikon COOLPIX P100) to quantify the amount of tissue consumed by the larvae. Leaf area was measured using the software ImageJ (Schneider et al., 2012). Data were analyzed using analyses of variance (ANOVA) and mean comparisons were performed through the Least Significant Difference (LSD) test $(p<0.05)$ conducted with the GLM procedure using the SAS software (SAS Institute, Cary, NC, United States).

\section{Glucosinolates Extraction and Quantification}

Sample extraction and desulfation, were performed according to Kliebenstein et al. (2001) with minor modifications. Ten microliters of the desulfo-GSL extract were used to identify and 
quantify the GSLs. The chromatographic analyses were carried out on an Ultra-High-Performance Liquid-Chromatograph (UHPLC Nexera LC-30AD; Shimadzu Corporation, Kyoto, Japan) equipped with a Nexera SIL-30AC injector and one SPD-M20A UV/VIS photodiode array detector. The UHPLC column was XSelect HSS T3 XP ColumnC18 protected with a C18 guard cartridge. The oven temperature was set at $30^{\circ} \mathrm{C}$. Compounds were separated using the following method in aqueous acetonitrile, with a flow of $0.5 \mathrm{~mL} \mathrm{~min}^{-1}: 1.5 \mathrm{~min}$ at $100 \% \mathrm{H}_{2} \mathrm{O}$, an $11 \mathrm{~min}$ gradient from 0 to $25 \%(\mathrm{v} / \mathrm{v})$ acetonitrile, $1.5 \mathrm{~min}$ at $25 \%(\mathrm{v} / \mathrm{v})$ acetonitrile, a minute gradient from 25 to $0 \%(\mathrm{v} / \mathrm{v})$ acetonitrile, and a final $3 \mathrm{~min}$ at $100 \% \mathrm{H}_{2} \mathrm{O}$. Data was recorded on a computer with the LabSolutions software (Shimadzu, Corporation, Kyoto, Japan). All GSLs were quantified at $229 \mathrm{~nm}$ by using glucotropaeolin (GTP, monohydrate from Phytoplan Diehm \& Neuberger GmbH, Heidelberg, Germany) as internal standard and quantified by comparison to purified standards. We reported the concentration (nmol per $g$ of DW) of individual GSL compounds as well as the sums of total aliphatic and indolic GSLs, two classes of GSLs based on the amino acid from which they have derived. ANOVA was used to compare GSL traits variation between control and treated plants by the GLM procedure using the SAS software (SAS Institute, Cary, NC, United States).

\section{Untargeted Metabolomics Analysis}

Extraction was performed using $20 \mathrm{mg}$ of lyophilized powder with $1 \mathrm{ml}$ of $80 \%$ aqueous methanol and vortexed for $10 \mathrm{~s}$ for homogenization. Extracts were sonicated in an ultrasonic bath (3510E-MTH, Bransonic ${ }^{\circledR}$, Mexico) for $5 \mathrm{~min}$ at $30 \mathrm{~Hz}$ frequency and then centrifuged for $10 \mathrm{~min}$ at maximum speed $(20,000 \times g)$ at room temperature. The supernatant was transferred to a clean tube. To extract the maximum of metabolites, a second extraction was performed, starting with the pellet and operating similarly as mentioned above, $200 \mu \mathrm{l}$ from the resulting supernatant were filtered using a 0.22 -micrometer micropore PTTE filter and placed in HPLC vials containing $0.8 \mathrm{ml}$ of $80 \%$ aqueous methanol. Five microliters of each sample were injected into an ultra-highperformance liquid chromatography (UHPLC) system (Thermo Dionex Ultimate 3000 LC) connected to a QTOF detector (Bruker Compact $^{\mathrm{TM}}$ ) with a heated electrospray ionization (ESI) source. Chromatographic separation was performed in a Bruker UHPLC Intensity Solo $2 \mathrm{C} 182.1 \times 100 \mathrm{~mm} 1.7 \mu \mathrm{m}$ pore size column using a binary gradient solvent mode, consisting of $0.1 \%$ formic acid in water (solvent $\mathrm{A}$ ) and acetonitrile (solvent $\mathrm{B})$. The following gradient was used: $3 \% \mathrm{~B}(0-3 \mathrm{~min})$, from 3 to $25 \% \mathrm{~B}(3-10 \mathrm{~min})$, from 25 to $80 \% \mathrm{~B}(10-18 \mathrm{~min})$, from 80 to $100 \% \mathrm{~B}(18-22 \mathrm{~min})$, and held at $100 \% \mathrm{~B}$ until $24 \mathrm{~min}$. The flow rate was established at $0.3 \mathrm{~mL} \mathrm{~min}-1$ and the column temperature was controlled at $35^{\circ} \mathrm{C}$. MS data were acquired using an acquisition rate of $2 \mathrm{~Hz}$ over the mass range of $50-1,200 \mathrm{~m} / \mathrm{z}$. Both polarities $( \pm)$ of ESI mode were used under the following specific conditions: gas flow $8 \mathrm{~L} \mathrm{~min}^{-1}$; nebulizer pressure 38 psi; dry gas $9 \mathrm{~L} \mathrm{~min}^{-1}$; dry temperature $220^{\circ} \mathrm{C}$. Capillary and end plate offset were set to 4,500 and $500 \mathrm{~V}$, respectively. LC-qTOF system stability was tested by three consecutive injections of chloramphenicol (ESI - mode;
$\Delta \mathrm{RT}=0.01 \mathrm{~min} ; \Delta \mathrm{m} / \mathrm{z}=0.002)$ and triphenyl phosphate (ESI + mode; $\Delta \mathrm{RT}=0.02 \mathrm{~min} ; \Delta \mathrm{m} / \mathrm{z}=0.001) . \mathrm{MS} / \mathrm{MS}$ analysis was performed in pooled samples (per day and condition). This analysis was operated in a spectrum acquisition range from 50 to $1,200 \mathrm{~m} / \mathrm{z}$ using an acquisition rate of $8 \mathrm{~Hz}$. Ions were targeted based on the previously determined accurate mass and retention time (RT) and fragmented by using different collision energy ramps to cover a range from 15 to $50 \mathrm{eV}$. Raw data from the UHPLC-QTOF were uploaded and processed by the commercial software MetaboScape 4.0 from Bruker (Kessler et al., 2016). Peak detection and data alignment were proceeded automatically using the T-ReX 3D algorithm. The resulted features matrix was imported to MetaboAnalyst (Chong et al., 2019). Data were normalized through Pareto scaling to avoid unwanted systemic biases (reducing the masking effect from abundant metabolites) with conserving data structure, keeping it partially intact (van den Berg et al., 2006; Yang et al., 2015). We performed two statistical analysis (multivariate and univariate analysis) to identify the most relevant features in the separation between the wound and control group of plants. These two methodologies may provide complementary biomarkers that could help to give a general overview of metabolic changes occurring in the plants (Saccenti et al., 2014). Multivariate analysis was performed using supervised partial least squares discriminate analysis (PLSDA) methodology. Selection of more discriminant features was performed using a threshold of VIP (variable importance in projection) score $>2$. Univariate analysis was performed using a Student $t$-test. To control the proportion of false positives among significant results that can be obtained due to multiple hypothesis testing we used false discovery rate (FDR $\leq 0.05)$. Features with an FDR $\leq 0.05$ and a $\mid \log 2 \mathrm{FC}$ (fold-change) $\mid \geq 1$ were considered differentially regulated. Metabolite identification was performed based on the exact mass and MS/MS spectrum. The software MetaboScape 4.0 (Bruker Daltonics, Germany) and SIRIUS 4 (Dührkop et al., 2019) were used to elucidate the metabolite molecular formula with default parameters. Both software use the exact mass and isotopic pattern to calculate the most probable molecular formula for each compound. In this analysis, only $\mathrm{C}, \mathrm{H}, \mathrm{O}, \mathrm{N}, \mathrm{S}$, and $\mathrm{P}$ were allowed to determine the molecular formula. Formulas with a mass deviation $<5 \mathrm{ppm}$ and a mSigma $<20$ (MetaboScape) were considered for annotation. For a tentative identification, publicly available metabolites databases were used [PubChem (Kim et al., 2021), KEGG (Kanehisa et al., 2021), CheBi (Hastings et al., 2016), ChemSpider (Pence and Williams, 2010), Metlin (Guijas et al., 2018)].

\section{RESULTS}

\section{Time of Day Contributes to Differential Phytochemical Responses to Mechanical Wounding}

We found significant variation among control and treated Brassica plants for individual and total GSLs (Figure 2). Our work revealed that changes on these defense-related compounds levels was dependent upon the time of day at which wounding was 


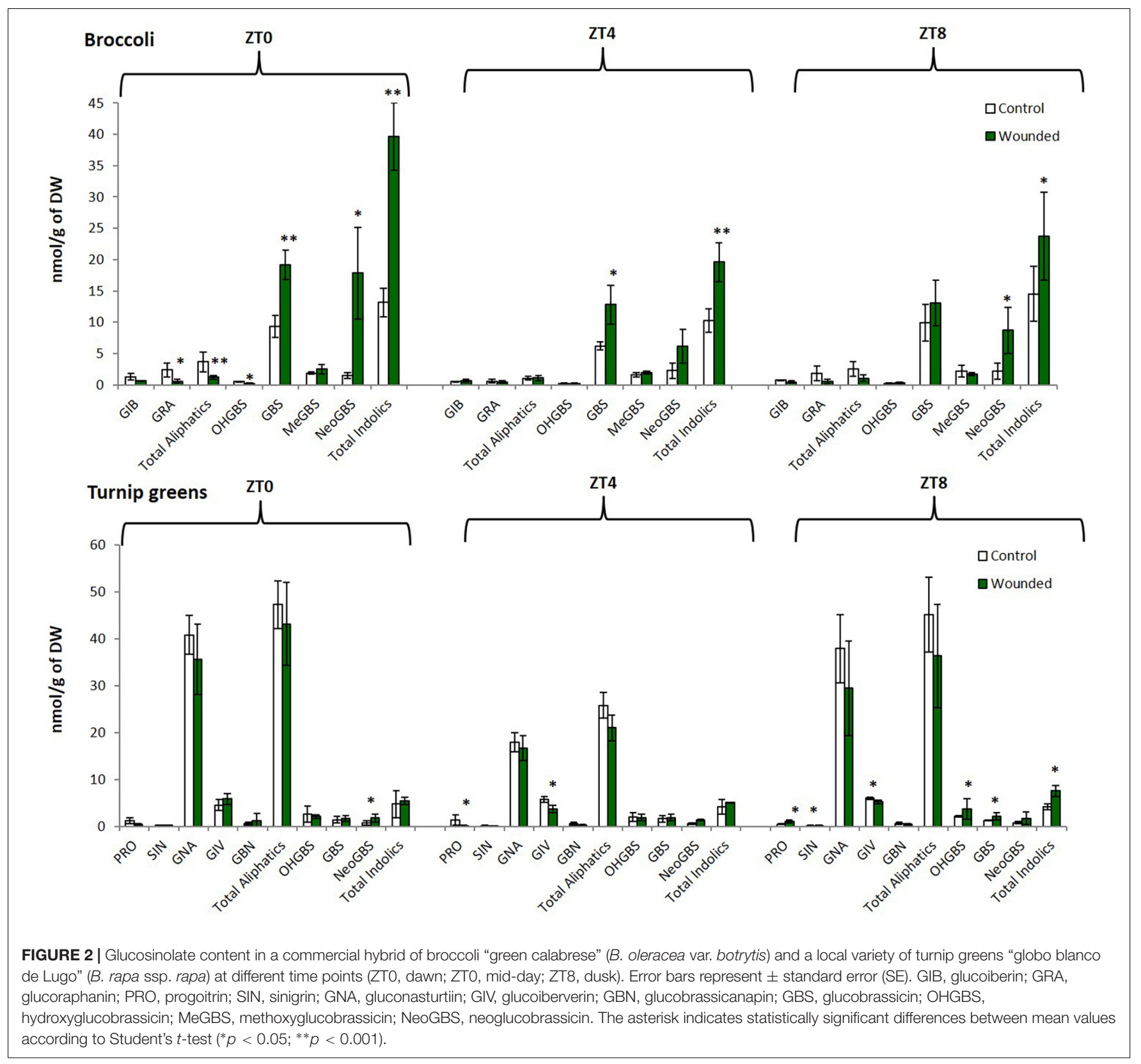

performed, and this response varied among species. Specifically, for broccoli the greatest changes on GSLs levels were found when wounding occurred at ZT0 (Figure 2). At this time point total and individual indolic GSLs were highly induced, for instance the content of Neoglucobrassicin (N-methoxy-3indolyl glucosinolate) from wounded plants was about 12 -fold higher than control. In contrast, aliphatic GSLs significantly decreased in broccoli (B. oleracea) plants wounded at ZT0 while aliphatic GSLs did not change when wounding occurred at the other time-points. For turnip greens ( $B$. rapa), most of the significant differences in GSLs traits were found when wounding occurred at ZT8 and the trend were similar to that found for broccoli, indole GSLs increased after wounding and aliphatic GSLs tend to decrease (Figure 2). These results indicate that the wound activation of distinct sets of defense metabolites is clock influenced in Brassica.

\section{Differential Plant Defense Response to the Time of Wounding Can Alter Mamestra brassicae Feeding Behavior}

To test whether the time-dependent accumulation of GSLs after wounding could also prime plants against insect feeding we performed a bioassay. In spite of different accumulation of GSLs in response to wounding, we only observed significant differences on larvae intake (measured as remained leaf area) among control and treated broccoli leaves at ZT0 $(P$-value $=0.0079)$ (Figure 3). For turnip greens, differences for larval food intake 

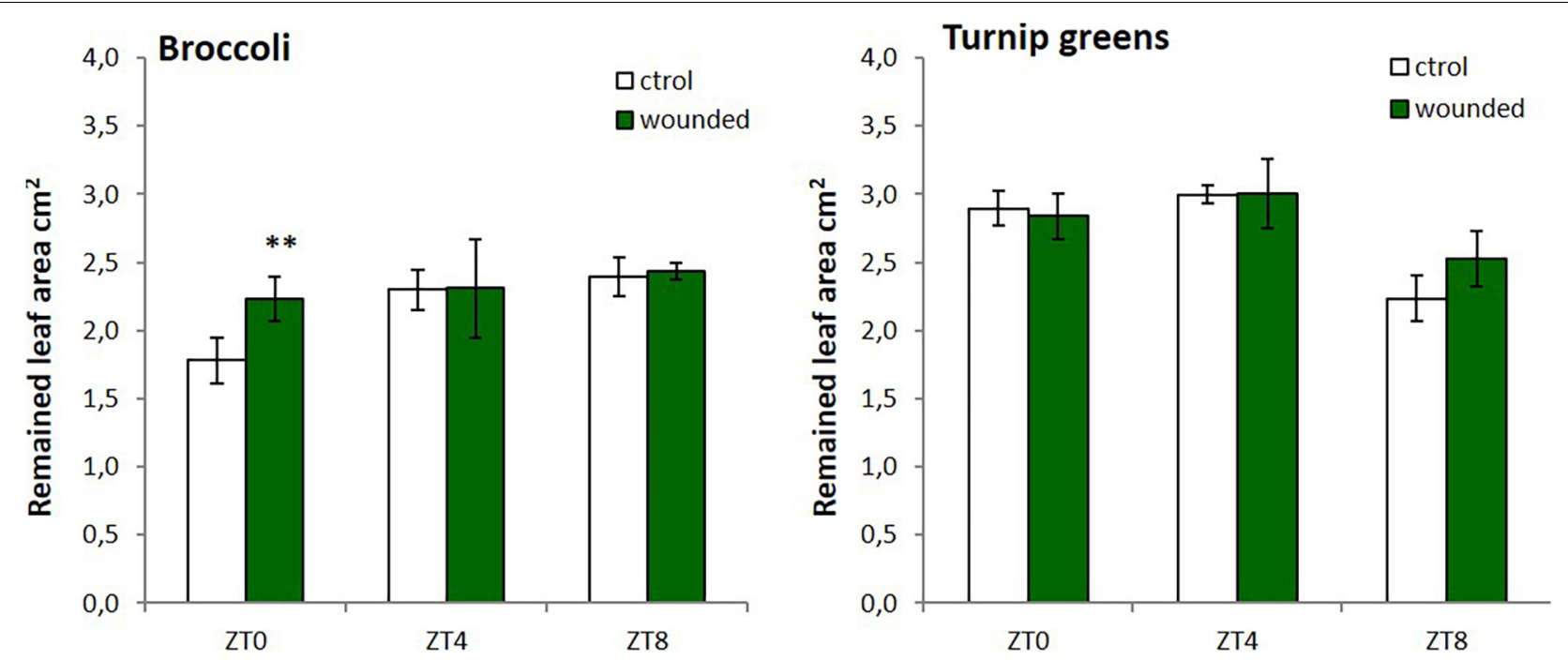

FIGURE 3 | Average area of leaves disk remaining after $24 \mathrm{~h}$ of Mamestra brassicae L. feeding. Error bars represent \pm standard error (SE). Leaves were collected from plants wounded at dawn (ZTO), mid-day (ZT4), or dusk (ZT8) during three consecutive days. The asterisk indicates statistically significant differences between mean values according to Student's $t$-test $\left({ }^{* *} p<0.001\right)$.

were found at ZT8, although it was not statistically significant $(P$-value $=0.053)$. This trend was not observed at other time points (Figure 3). Thus, maximal responsiveness of plant defense against $M$. brassicae is stimulated by wounding at ZT0 in broccoli and at ZT8 in turnip greens. These results indicate that the effect of the time-dependent wounding on herbivory can only partly be mediated by GSLs and that other factors must be involved in the response.

\section{Identifying Plant Metabolites Involved in Brassica Defense Using an Untargeted Metabolomics Approach}

To define a comprehensive landscape of metabolite reprogramming underlying time-of-day induced resistance of Brassica crops, we carried out an untargeted metabolomics analysis using UPLC-Q-TOF-MS/MS. We analyzed global trends of metabolite variations by searching for changes that occur in leaves from ZT0 and ZT8 wound-entrained plants compared with control plants for broccoli and turnip greens, respectively. We focused on ZT0 and ZT8 because they were the samples with larval food intake differences. A total of 466 features in broccoli leaves and 619 features in turnip greens leaves were detected. The PLS-DA score plot shows no discrimination between samples for turnip greens, indicating that wounding entrainment at ZT8 had no visible effect on the global metabolite profiles of wounded plants. We did, however, find a discernible metabolite separation between control and unwounded plants at ZT0 in the broccoli leaves. Therefore, further analysis and metabolite annotation are referred to broccoli samples.

Among the 466 ions significantly altered in broccoli, 47 were selected as important features (22 based on VIPscore values of the PLS-DA analysis and 25 based on the volcano-plot analysis) contributing to the ZT0 wound response
(Supplementary Table 1). A hierarchical cluster analysis grouped these 47 metabolites into two separate groups (Figure 4A). One group encompasses metabolites with a lower accumulation in unwounded leaves of plants wounded at ZT0 compared to that of the control plants. We were unable to assign a candidate compound to most of these compounds (Figure 4B). The other group encompasses metabolites with higher accumulation in unwounded leaves of plants wounded at ZT0. Most of these compounds are classified as indoles or indole derivates or isothiocyanates (Figure 4C) based on the ClassyFire chemical taxonomy (Djoumbou Feunang et al., 2016).

\section{DISCUSSION}

Circadian clock regulation of stress signaling allows the plant to prepare for unpleasant events and to gate suitable responses in a time-of-day manner. Our results show a direct contribution of time of day on the ability of wounding to systemically induce specialized metabolism of Brassica crops. Within a fixed light/dark cycle, plant defense response to wounding elicitation depended on the diurnal timing of the wounding (Figure 2). Induction of indole GSLs at dawn for broccoli and dusk for turnip greens was 2-12 times higher when compared with wounding during the day. Remarkable, the time-of-day response of GSLs varied by genotype. Previous work has also shown that wound or other herbivory responses vary across species and genotypes in their circadian or diurnal response. Arimura et al. (2008) showed that nocturnal damage to lima bean leaves increased JA levels 2-3 times higher when compared with day damage; In contrast, sensitivity to JA in Arabidopsis plants is highest at dawn (Baldwin and Meldau, 2013). In Nicotiana attenuata, the peak accumulation of several herbivory-induced defense metabolites, after simulated herbivory varies between 


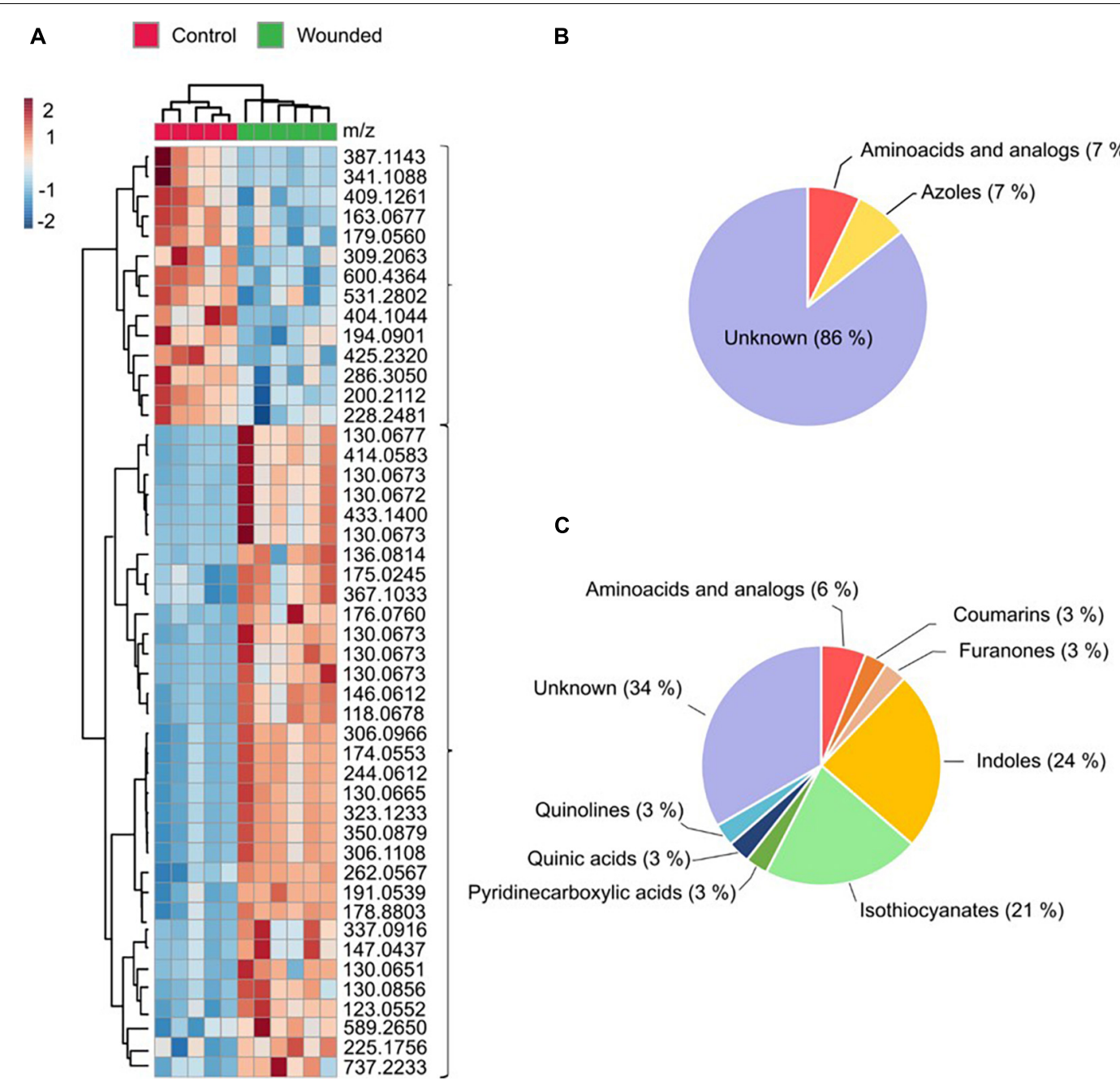

FIGURE 4 | Identification of metabolites involved in the wounding defensive response in broccoli (B. oleracea var. botrytis). Samples were harvested at dawn (ZTO). (A) Cluster heatmap analysis of the intensity of 47 metabolites differentially accumulated in response to wounding. (B) Grouping of cluster of metabolites with lower accumulation in wounded plants based on the ClassyFire chemical taxonomy. (C) Grouping of cluster of metabolites with higher accumulation in wounded plants based on the ClassyFire chemical taxonomy. The scale indicates the color code relative to the normalized metabolite abundance (ranging from -2 up to 2 ).

local and systemic tissues, leaves and roots (Kim et al., 2011). Natural variation on diurnal and/or circadian regulation of plant defense is likely to have ecological and breeding significance because plants may have been selected to maximize its antiherbivore defenses to the time-of-day that is most valuable or vulnerable to attack. Therefore, we hypothesized the existence of interconnections among temporal induction of specialized metabolites and herbivore performance.

The Lepidopteran insect $M$. brassicae is a generalist insect that feeds on plants from several species belonging to more than 20 families, of which members of the Brassicaceae are among the most preferred (Rojas et al., 2000; Cartea et al., 2009). Feeding by the caterpillars causes severe damage to the plants, and it is an economically devastating pest in agriculture (Cartea et al., 2010). Although it is documented that the moth of this insect is most active at night (Devetak et al., 2010), we found that at the larvae stage, under constant temperature conditions they feed similarly during light and dark periods. Hence, any change in the feeding preference of larva under the same conditions could be attributed to the differential chemical composition of the plant. Earlier reports have been shown that $M$. brassicae preference and performance are both affected by GSLs content and composition when comparing genotypes in choice and non-choice feeding experiments (Cartea et al., 2010; Jeschke et al., 2017; BadenesPérez and Cartea, 2021). However, how wounding and time-ofday may prime the plant to influence herbivore performance has not been previously reported. Here we demonstrated that the time of wounding alters $M$. brassicae feeding behavior in time and genotype-dependent manner. M. brassicae caterpillars consumed less leaf material when broccoli was wounded at ZT0 while in turnip greens the lowest consumption was found in plants wounded at ZT8. This correlates with the treatment that maximized GSL induction accumulation in each crop (Figures 2, 3). This suggests that plants may be adapted to use the 


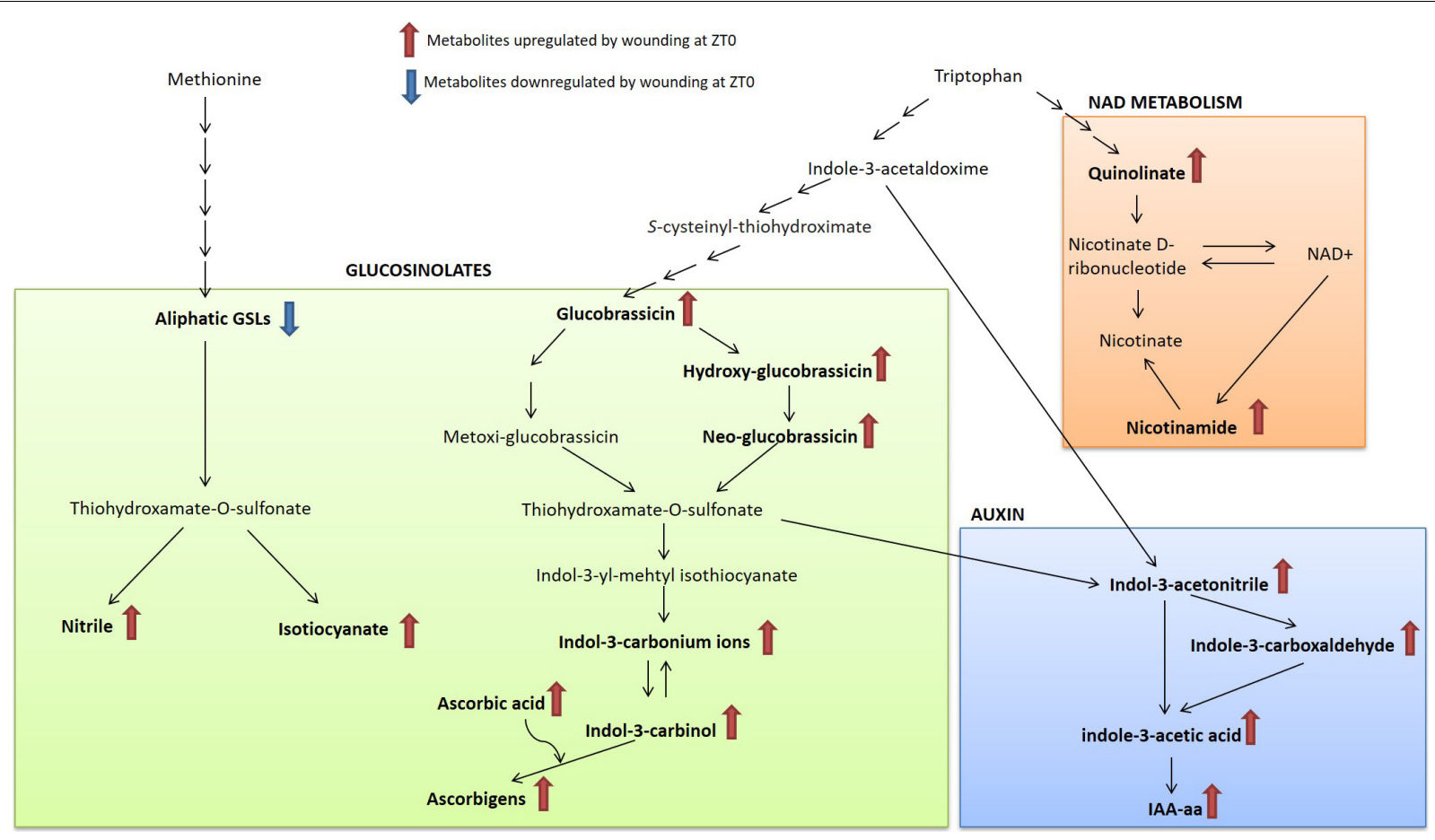

FIGURE 5 | Schematic representation of the major metabolic pathways activated in response to wounding in broccoli (B. oleracea var. botrytis). Metabolites in bold show a significant variation after wounding treatment. NAD, nicotinamide adenine dinucleotide; IAA-aa, Indole-3-acetic acid conjugated by amide linkage to amino acids.

circadian clock to schedule and gate defense to match the likely timing of an attack under natural conditions.

Further dissection of the metabolic reconfiguration shed some light on the time-of-day influence over wound induced defense network of broccoli plants against $M$. brassicae. This analysis showed that GSLs breakdown products, IAA components, NAD intermediates and pipecolic acid were the identified metabolites that most significantly contributed for the chemical induced response of broccoli at dawn (Figure 4 and Supplementary Table 1). In turnip greens, there was no clear effect of wounding on the global metabolite profiles.

Upon tissue damage, such as herbivory or wounding, GSL come into contact with myrosinase enzyme (thioglucoside glucohydrolase EC 3.2.1.147). The hydrolysis originates an unstable intermediary (thiohydroxamate- $O$-sulfonate) that decomposes into a series of products such as ITCs, nitriles, thiocyanates, epithionitriles and oxazolidines depending on the chemical nature of the parent GSL, the presence of other different enzymes, the $\mathrm{pH}$ and cofactors (Wittstock and Halkier, 2002). Metabolomics analysis reveals that ITCs, nitriles and other downstream bioactive compounds were up-regulated in broccoli wounded leaves at morning (Figures 4, 5). The ITCs are highly reactive and can react with water, ascorbate, glutathione, amino acids, and other plant metabolites to produce a variety of physiologically active compounds associated with herbivore and pathogen defense (i.e., indole-3-carbinol) (Clay et al., 2009). On the contrary, the nitrile formation pathway has been proposed as a possible route of non-toxic GSLs turnover to rebuild primary metabolites (Kissen and Bones, 2009), although recently they seem to be involved into a coordinated crosstalk with phytohormone signaling (Katz et al., 2020; Ting et al., 2020). Over all, our results indicated that the plant may be more prone to trigger a GSL-related defense response when wounding occurs during the morning. Supporting this notion, Brandt et al. (2018) observed a diurnal rhythm for myrosinase activity with a significant increase of activity at the beginning of the light period in Arabidopsis. Further research is necessary to dissect the direct connections between GSL catabolism and the clock and test whether this response can be extended to other Brassica crop species.

Interestingly, the growth regulator IAA, a natural auxin, has been shown to be up-regulated in response to wounding (Figure 5). IAA is necessary for the proper development of embryos, roots, and shoots, and is also well known for its role in gravitropism and phototropism (Ludwig-Müller, 2011). Early studies reported an apparent antagonism between IAA and plant immunity against wounding and/or herbivory (Erb et al., 2012; Casalongué et al., 2014; Machado et al., 2016). This is explained for the involvement of auxins in the trade-off between growth and defense (Naseem et al., 2015). However, here we demonstrated that broccoli plants entrained to receive an attack during the morning increased their IAA levels altogether with specialized defensive compounds. This indicates that plants may have different strategies depending on the time of day at which damage occurs. This potential is supported by recent research suggesting that IAA accumulation is a rapid and specific signal that 
regulates a subset of systemic, hormone-dependent secondary metabolites under herbivore-attacked plants (Machado et al., 2016). Supporting this hypothesis, we also detected a possible relationship between GSL catabolism and IAA homeostasis in response to wounding.

One possible connection between GSL catabolism and IAA homeostasis is that indolic GSL can catabolize to indole-3acetonitrile and then further converted into auxin, and all the intermediary metabolites of this pathway were up-regulated under wounding stress at dawn in broccoli (Figure 5). A tendency of IAA accumulation in accordance with turnover of indolic GSLs has been previously observed (Müller and Weiler, 2000; Reintanz et al., 2001). It has been suggested that this pathway is likely to be operative only under special environmental circumstances (Malka and Cheng, 2017). However, the physiological role of this interaction is so far not conclusive. The fact that promotion of auxin accumulation in coordination with GSL breakdown product reduced the herbivore preference of wounded leaves, strongly suggest that GSL-auxin homeostasis is an important determinant of broccoli tolerance against herbivory. A deeper knowledge of this response would allow optimizing cultural practices against pests to optimize plant fitness in the field.

A closer examination of untargeted metabolomic analyses revealed that other metabolites, such as nicotinamide and pipecolate, critical regulators of inducible plant immunity (Návarová et al., 2013; Miwa et al., 2017; Sidiq et al., 2021) as well as the naturally occurring sulfur compound, SMCSO, were also involved on the broccoli defense priming (Figure 5). Even though these metabolites appear to act as modulators in the coordination of defense responses against biotrophic and necrotrophic pathogens, here we report that they may serve as novel priming agent for insect pest control in crop production. Results from this work will facilitate future research investigating how specialized metabolites respond to a dieldependent repetitive stress.

As a result of millions of years of co-evolution plants have evolved sophisticated mechanism to anticipate and protect themselves against insect attack. Our results indicate that the daily rhythmicity of insect feeding allow the plant to accumulate defensive compounds prior to the highest peaks of insect activity. The fine-tuning of this response is reflected in the differential response observed among species. At the molecular level, the preventive response involved mainly accumulation of GSLs and related compounds along with indoles involved in the biosynthesis of auxins.

\section{REFERENCES}

Agrawal, A. A., Conner, J. K., Johnson, M. T. J., and Wallsgrove, R. (2002). Ecological genetics of an induced plant defense against herbivores: additive genetic variance and costs of phenotypic plasticity. Evolution 56, 2206-2213. doi: 10.1111/j.0014-3820.2002.tb00145.x

Arimura, G. I., Köpke, S., Kunert, M., Volpe, V., David, A., Brand, P., et al. (2008). Effects of feeding Spodoptera littoralis on lima bean leaves: IV. Diurnal and nocturnal damage differentially initiate plant volatile emission. Plant Physiol. 146, 965-973. doi: 10.1104/pp.107.111088

\section{DATA AVAILABILITY STATEMENT}

The original contributions presented in the study are included in the article/Supplementary Material, further inquiries can be directed to the corresponding author/s.

\section{AUTHOR CONTRIBUTIONS}

MF and VR conceived and designed the experiments. MD, MF, and VR assisted with the plant trials setup, conducted the laboratory work, and did the metabolomics analysis. MD, MF, DK, and VR analyzed the data and wrote the manuscript. All authors read and approved the final manuscript.

\section{FUNDING}

This effort was funded by the grants RTI2018-094650-J-I00 and RYC2019-027834-I by the MCIN/AEI/10.13039/501100011033 and "ESF Investing in your future" to MF.

\section{SUPPLEMENTARY MATERIAL}

The Supplementary Material for this article can be found online at: https://www.frontiersin.org/articles/10.3389/fpls.2021. 807710/full\#supplementary-material

Supplementary Figure 1 | (A) Multivariate analysis using supervised partial least squares discriminate analysis (PLS-DA) in separation of control and wounded leaves from broccoli leaves harvested at ZTO. (B) Cross validation analysis indicated that two components model was the optimal model for broccoli samples. (C) PLS-DA analysis of control and wounded leaves from turnip greens harvested at ZT8. (D) Cross validation analysis indicated no discrimination between turnip greens samples. Colored circles represent $95 \%$ confidence intervals. Colored dots represent individual samples. The component that best classifies the model is shown with red asterisk.

Supplementary Figure 2 | Volcano plot displaying features that were most differentially expressed at wounded broccoli plants relative to control at ZTO.

Supplementary Table 1 | Metabolomic dataset of features detected in broccoli leaves using UPLC-Q-TOF-MS/MS.

Supplementary Table 2 | Metabolomic dataset of features detected in turnip greens leaves using UPLC-Q-TOF-MS/MS.

Supplementary Table 3 | Metabolomic dataset of important features (based on VIP-score values of the PLS-DA analysis and on the volcano-plot analysis) contributing to the responses of wounding and $M$. brassicae feeding behavior from broccoli samples at ZTO.

Badenes-Pérez, F. R., and Cartea, M. E. (2021). Glucosinolate induction and resistance to the cabbage moth, mamestra brassicae, differs among kale genotypes with high and low content of sinigrin and glucobrassicin. Plants 10:1951. doi: 10.3390/plants10091951

Baldwin, I. T., and Meldau, S. (2013). Just in time. Plant Signal. Behav. 8:e24410. doi: $10.4161 /$ psb. 24410

Bhardwaj, V., Meier, S., Petersen, L. N., Ingle, R. A., and Roden, L. C. (2011). Defence responses of arabidopsis thaliana to infection by Pseudomonas syringae are regulated by the circadian clock. PLoS One 6:e26968. doi: 10.1371/journal. pone.0026968 
Body, M. J. A., Neer, W. C., Vore, C., Lin, C. H., Vu, D. C., Schultz, J. C., et al. (2019). Caterpillar chewing vibrations cause changes in plant hormones and volatile emissions in Arabidopsis thaliana. Front. Plant Sci. 10:810. doi: 10.3389/ FPLS.2019.00810

Brandt, S., Fachinger, S., Tohge, T., Fernie, A. R., Braun, H. P., and Hildebrandt, T. M. (2018). Extended darkness induces internal turnover of glucosinolates in Arabidopsis thaliana leaves. PLoS One 13:e0202153. doi: 10.1371/journal.pone. 0202153

Burow, M., and Halkier, B. A. (2017). How does a plant orchestrate defense in time and space? Using glucosinolates in Arabidopsis as case study. Curr. Opin. Plant Biol. 38, 142-147. doi: 10.1016/j.pbi.2017.04.009

Cartea, M. E., Francisco, M., Lema, M., Soengas, P., and Velasco, P. (2010). Resistance of cabbage (Brassica oleracea capitata Group) crops to Mamestra brassicae. J. Econ. Entomol. 103, 1866-1874. doi: 10.1603/EC09375

Cartea, M. E., Padilla, G., Vilar, M., and Velasco, P. (2009). Incidence of the major Brassica pests in Northwestern Spain. J. Econo. Entomol. 102, 767-773. doi: 10.1603/029.102.0238

Casalongué, C. A., Fiol, D. F., París, R., Godoy, A. V., D’Ippólito, S., and Terrile, M. C. (2014). "Auxin as part of the wounding response in plants," in Phytohormones and Abiotic Stress Tolerance in Plants, eds N. A. Khan, R. Nazar, N. Iqbal, and N. A. Anjum (Berlin: Springer-Verlag), doi: 10.1007/978-3-64225829-9_5

Chong, J., Wishart, D. S., and Xia, J. (2019). Using metaboanalyst 4.0 for comprehensive and integrative metabolomics data analysis. Curr. Protocols Bioinformatics 68:e86. doi: 10.1002/cpbi.86

Clay, N. K., Adio, A. M., Denoux, C., Jander, G., and Ausubel, F. M. (2009). Glucosinolate metabolites required for an Arabidopsis innate immune response. Science 323, 95-101. doi: 10.1126/science.1164627

Covington, M. F., Maloof, J. N., Straume, M., Kay, S. A., and Harmer, S. L. (2008). Global transcriptome analysis reveals circadian regulation of key pathways in plant growth and development. Genome Biol. 9:R130. doi: 10.1186/gb-2008-98-r130

Devetak, M., Vidrih, M., and Trdan, S. (2010). Cabbage moth (Mamestra brassicae [L.]) and bright-line brown-eyes moth (Mamestra oleracea [L.]) - presentation of the species, their monitoring and control measures. Acta Agric. Slovenica 95, 149-156. doi: 10.2478/v10014-010-0011-3

Djoumbou Feunang, Y., Eisner, R., Knox, C., Chepelev, L., Hastings, J., Owen, G., et al. (2016). ClassyFire: automated chemical classification with a comprehensive, computable taxonomy. J. Cheminform. 8:61. doi: 10.1186/ s13321-016-0174-y

Dührkop, K., Fleischauer, M., Ludwig, M., Aksenov, A. A., Melnik, A. V., Meusel, M., et al. (2019). SIRIUS 4: a rapid tool for turning tandem mass spectra into metabolite structure information. Nat. Methods 16, 299-302. doi: 10.1038/ s41592-019-0344-8

Erb, M., Meldau, S., and Howe, G. A. (2012). Role of phytohormones in insectspecific plant reactions. Trends Plant Sci. 17, 250-259. doi: 10.1016/j.tplants. 2012.01.003

Goodspeed, D., Chehab, E. W., Min-Venditti, A., Braam, J., and Covington, M. F. (2012). Arabidopsis synchronizes jasmonate-mediated defense with insect circadian behavior. Proc. Natl. Acad. Sci. U.S. A. 109, 4674-4677. doi: 10.1073/ pnas. 1116368109

Goodspeed, D., Liu, J. D., Chehab, E. W., Sheng, Z., Francisco, M., Kliebenstein, D. J., et al. (2013). Postharvest circadian entrainment enhances crop pest resistance and phytochemical cycling. Curr. Biol. 23, 1235-1241. doi: 10.1016/j. cub.2013.05.034

Guan, Y., Hu, W., Xu, Y., Yang, X., Ji, Y., Feng, K., et al. (2021). Metabolomics and physiological analyses validates previous findings on the mechanism of response to wounding stress of different intensities in broccoli. Food Res. Int.l 140:110058. doi: 10.1016/J.FOODRES.2020.110058

Guijas, C., Montenegro-Burke, J. R., Domingo-Almenara, X., Palermo, A., Warth, B., Hermann, G., et al. (2018). METLIN: a technology platform for identifying knowns and unknowns. Anal. Chem. 90, 3156-3164. doi: 10.1021/ACS. ANALCHEM.7B04424

Harmer, S. L., Hogenesch, J. B., Straume, M., Chang, H. S., Han, B., Zhu, T., et al. (2000). Orchestrated transcription of key pathways in Arabidopsis by the circadian clock. Science 290, 2110-2113. doi: 10.1126/science.290.5499.2110

Hastings, J., Owen, G., Dekker, A., Ennis, M., Kale, N., Muthukrishnan, V., et al. (2016). ChEBI in 2016: improved services and an expanding collection of metabolites. Nucleic Acids Res. 44, D1214-D1219. doi: 10.1093/NAR/ GKV1031

Jacobo-Velázquez, D. A., González-Aguëro, M., and Cisneros-Zevallos, L. (2015). Cross-talk between signaling pathways: the link between plant secondary metabolite production and wounding stress response. Sci. Rep. 5:8608. doi: 10.1038/srep08608

Jansen, J. J., Allwood, J. W., Marsden-Edwards, E., van der Putten, W. H., Goodacre, R., and van Dam, N. M. (2009). Metabolomic analysis of the interaction between plants and herbivores. Metabolomics 5, 150-161. doi: 10 . 1007/s11306-008-0124-4

Jeschke, V., Kearney, E. E., Schramm, K., Kunert, G., Shekhov, A., Gershenzon, J., et al. (2017). How glucosinolates affect generalist lepidopteran larvae: growth, development and glucosinolate metabolism. Front. Plant Sci. 8:1995. doi: 10. 3389/fpls.2017.01995

Kanehisa, M., Furumichi, M., Sato, Y., Ishiguro-Watanabe, M., and Tanabe, M. (2021). KEGG: integrating viruses and cellular organisms. Nucleic Acids Res. 49, D545-D551. doi: 10.1093/NAR/GKAA970

Katz, E., Bagchi, R., Jeschke, V., Rasmussen, A. R. M., Hopper, A., Burow, M., et al. (2020). Diverse allyl glucosinolate catabolites independently influence root growth and development. Plant Physiol. 183, 1376-1390. doi: 10.1104/PP.20. 00170

Kessler, N., Neuweger, H., Tellström, V., and Barsch, A. (2016). What Are We Eating? MetaboScape ${ }^{\circledR}$ Software; Enabling the De-replication and Identification of Unknowns in Food Metabolomics. Bremen: Bruker Daltonics.

Kim, S., Chen, J., Cheng, T., Gindulyte, A., He, J., He, S., et al. (2021). PubChem in 2021: new data content and improved web interfaces. Nucleic Acids Res. 49, D1388-D1395. doi: 10.1093/NAR/GKAA971

Kim, S.-G., Yon, F., Gaquerel, E., Gulati, J., and Baldwin, I. T. (2011). Tissue specific diurnal rhythms of metabolites and their regulation during herbivore attack in a native tobacco Nicotiana attenuata. PLoS One 6:e26214. doi: 10.1371/journal. pone.0026214

Kissen, R., and Bones, A. M. (2009). Nitrile-specifier proteins involved in glucosinolate hydrolysis in Arabidopsis thaliana. J. Biol. Chem. 284, 12057 12070. doi: 10.1074/jbc.M807500200

Kliebenstein, D. J., Gershenzon, J., and Mitchell-Olds, T. (2001). Comparative quantitative trait loci mapping of aliphatic, indolic and benzylic glucosinolate production in Arabidopsis thaliana leaves and seeds. Genetics 159, 359-370. doi: 10.1093/genetics/159.1.359

Lei, J., and Zhu-Salzman, K. (2021). Late elongated hypocotyl potentiates resistance conferred by circadian clock associated 1 to aphid by co-regulating the expression of indole glucosinolate biosynthetic genes. Plant Signal. Behav. 16:1908708. doi: 10.1080/15592324.2021.1908708

Lei, J., Jayaprakasha, G. K., Singh, J., Uckoo, R., Borrego, E. J., Finlayson, S., et al. (2019). CIRCADIAN CLOCK-ASSOCIATED1 controls resistance to aphids by altering indole glucosinolate production. Plant Physiol. 18:1344. doi: 10.1104/ pp. 19.00676

Ludwig-Müller, J. (2011). Auxin conjugates: their role for plant development and in the evolution of land plants. J. Exp. Bot. 62, 1757-1773. doi: 10.1093/jxb/ erq412

Machado, R. A. R., Robert, C. A. M., Arce, C. C. M., Ferrieri, A. P., Xu, S., JimenezAleman, G. H., et al. (2016). Auxin is rapidly induced by herbivore attack and regulates a subset of systemic, jasmonate-dependent defenses. Plant Physiol. 172, 521-532. doi: 10.1104/pp.16.00940

Malka, S. K., and Cheng, Y. (2017). Possible interactions between the biosynthetic pathways of indole glucosinolate and Auxin. Front. Plant Sci. 8:2131. doi: 10 . 3389/fpls.2017.02131

McClung, C. R. (2019). The plant circadian oscillator. Biology 8:14. doi: 10.3390/ biology 8010014

Miwa, A., Sawada, Y., Tamaoki, D., Hirai, M. Y., Kimura, M., Sato, K., et al. (2017). Nicotinamide mononucleotide and related metabolites induce disease resistance against fungal phytopathogens in Arabidopsis and barley. Sci. Rep. 7:6389. doi: 10.1038/s41598-017-06048-8

Müller, A., and Weiler, E. W. (2000). Indolic constituents and indole-3-acetic acid biosynthesis in the wild-type and a tryptophan auxotroph mutant of Arabidopsis thaliana. Planta 211, 855-863. doi: 10.1007/s004250000353

Naseem, M., Kaltdorf, M., and Dandekar, T. (2015). The nexus between growth and defence signalling: auxin and cytokinin modulate plant immune response pathways. J. Exp. Bot. 66, 4885-4896. doi: 10.1093/jxb/erv297 
Návarová, H., Bernsdorff, F., Döring, A. C., and Zeier, J. (2013). Pipecolic acid, an endogenous mediator of defense amplification and priming, is a critical regulator of inducible plant immunity. Plant Cell 24, 5123-5141. doi: 10.1105/ tpc.112.103564

Paul-Victor, C., Züst, T., Rees, M., Kliebenstein, D. J., and Turnbull, L. A. (2010). A new method for measuring relative growth rate can uncover the costs of defensive compounds in Arabidopsis thaliana. New Phytol. 187, 1102-1111. doi: $10.1111 /$ j.1469-8137.2010.03325.x

Pence, H. E., and Williams, A. (2010). ChemSpider: an online chemical information resource. J. Chem. Educ. 87, 1123-1124. doi: 10.1021/ED100697W

Reintanz, B., Lehnen, M., Reichelt, M., Gershenzon, J., Kowalczyk, M., Sandberg, G., et al. (2001). bus, a bushy Arabidopsis CYP79F1 knockout mutant with abolished synthesis of short-chain aliphatic glucosinolates. Plant Cell 13, 351367. doi: 10.1105/tpc.13.2.351

Rojas, J. C., Wyatt, T. D., and Birch, M. C. (2000). Flight and oviposition behavior toward different host plant species by the cabbage moth, Mamestra brassicae (L.) (Lepidoptera: Noctuidae). J. Insect Behav. 13, 247-254. doi: 10.1023/A: 1007792332046

Saccenti, E., Hoefsloot, H. C. J., Smilde, A. K., Westerhuis, J. A., and Hendriks, M. M. W. B. (2014). Reflections on univariate and multivariate analysis of metabolomics data. Metabolomics 10, 361-374. doi: 10.1007/s11306-0130598-6

Savatin, D. V., Gramegna, G., Modesti, V., and Cervone, F. (2014). Wounding in the plant tissue: the defense of a dangerous passage. Front. Plant Sci. 5:470. doi: 10.3389/fpls.2014.00470

Schneider, C. A., Rasband, W. S., and Eliceiri, K. W. (2012). NIH Image to ImageJ: 25 years of image analysis. Nat. Methods 9, 671-675. doi: 10.1038/nmeth.2089

Sidiq, Y., Nakano, M., Mori, Y., Yaeno, T., Kimura, M., and Nishiuchi, T. (2021). Nicotinamide effectively suppresses fusarium head blight in wheat plants. Int. J. Mol. Sci. 2:2968. doi: 10.3390/ijms22062968

Soengas, P., Cartea, M. E., Velasco, P., and Francisco, M. (2018a). Brassica glucosinolate rhythmicity in response to light-dark entrainment cycles is cultivar-dependent. Plant Sci. 275, 28-35. doi: 10.1016/j.plantsci.2018.07.009

Soengas, P., Cartea, M. E., Velasco, P., and Francisco, M. (2018b). Endogenous circadian rhythms in polyphenolic composition induce changes in antioxidant properties in brassica cultivars. J. Agric. Food Chem. 66, 5984-5991. doi: 10. 1021/acs.jafc.8b01732

Ting, H. M., Cheah, B. H., Chen, Y. C., Yeh, P. M., Cheng, C. P., Yeo, F. K. S., et al. (2020). The role of a glucosinolate-derived nitrile in plant immune responses. Front. Plant Sci. 11:257. doi: 10.3389/fpls.2020.00257 van Dam, N. M., Witjes, L., and Svatoš, A. (2004). Interactions between aboveground and belowground induction of glucosinolates in two wild Brassica species. New Phytol. 161, 801-810. doi: 10.1111/j.1469-8137.2004.00984.x

van den Berg, R. A., Hoefsloot, H. C. J., Westerhuis, J. A., Smilde, A. K., and van der Werf, M. J. (2006). Centering, scaling, and transformations: improving the biological information content of metabolomics data. BMC Genomics 7:142. doi: 10.1186/1471-2164-7-142

Walley, J. W., Coughlan, S., Hudson, M. E., Covington, M. F., Kaspi, R., Banu, G., et al. (2007). Mechanical stress induces biotic and abiotic stress responses via a novel cis-element. PLoS Genet. 3:e172. doi: 10.1371/journal.pgen.00 30172

Wang, W., Barnaby, J. Y., Tada, Y., Li, H., Tor, M., Caldelari, D., et al. (2011). Timing of plant immune responses by a central circadian regulator. Nature 470, 110-114.

Wittstock, U., and Halkier, B. A. (2002). Glucosinolate research in the Arabidopsis era. Trends Plant Sci. 7, 263-270. doi: 10.1016/S1360-1385(02)02273-2

Yang, J., Zhao, X., Lu, X., Lin, X., and Xu, G. (2015). A data preprocessing strategy for metabolomics to reduce the mask effect in data analysis. Front. Mol. Biosci. 2:4. doi: 10.3389/fmolb.2015.00004

Zhang, C., Xie, Q., Anderson, R. G., Ng, G., Seitz, N. C., Peterson, T., et al. (2013). Crosstalk between the circadian clock and innate immunity in Arabidopsis. PLoS Pathog. 9:e1003370. doi: 10.1371/journal.ppat.1003370

Conflict of Interest: The authors declare that the research was conducted in the absence of any commercial or financial relationships that could be construed as a potential conflict of interest.

Publisher's Note: All claims expressed in this article are solely those of the authors and do not necessarily represent those of their affiliated organizations, or those of the publisher, the editors and the reviewers. Any product that may be evaluated in this article, or claim that may be made by its manufacturer, is not guaranteed or endorsed by the publisher.

Copyright (C) 2022 Doghri, Rodríguez, Kliebenstein and Francisco. This is an openaccess article distributed under the terms of the Creative Commons Attribution License (CC BY). The use, distribution or reproduction in other forums is permitted, provided the original author(s) and the copyright owner(s) are credited and that the original publication in this journal is cited, in accordance with accepted academic practice. No use, distribution or reproduction is permitted which does not comply with these terms. 